\title{
An Evaluation of the Roadwise Review: A Mixed Methods Approach
}

To cite this article: Michelle M. Porter \& Holly A. Tuokko (2011) An Evaluation of the Roadwise Review: A Mixed Methods Approach, Traffic Injury Prevention, 12:5, 451-458, DOI: 10.1080/15389588.2011.601371

To link to this article: https://doi.org/10.1080/15389588.2011.601371

Michelle M. Porter, BPHE, MSc, PhD (corresponding author)

Faculty of Kinesiology and Recreation Management

University of Manitoba

Winnipeg, MB Canada

R3T 2N2

(204) 474-8795

Fax: (204) 261-4802

portermm@cc.umanitoba.ca

Holly A. Tuokko, PhD, RPsych

Director, Centre on Aging

Sedgewick Building, Room A104

University of Victoria

PO Box 1700 STN CSC

Victoria, BC V8W 2Y2

(250) $721-6350$

Fax: (250) 721-6499

htuokko@uvic.ca 


\section{ABSTRACT}

Objective: The objective of this mixed methods study was to perform an evaluation of the Roadwise Review CD-ROM (RWR), a self-screening tool for older drivers.

Methods: Ninety-six older drivers (67 to 91 ) used the RWR with a research assistant at two different sites, and then completed written surveys (closed and open-ended questions) immediately after the session as well as two weeks later. In addition, RWR test results by these 96 older drivers were compared to the results of the original sample that was used to establish normative data for the RWR.

Results: When test scores were evaluated, almost all participants (96\%) were found to have at least one impairment. The rates of impairment varied dramatically between tests from $3 \%$ (visual acuity) to $75 \%$ (visual search). Several average test scores were found to be different from the original samples upon which the normative data were derived. Several factors were found that influenced these older drivers' scores on the various components of the RWR, although the explanatory power was very low. Participants reported that the RWR was useful, they made changes to their driving, and talked to their family and friends about driving. Participants also provided many good suggestions for improvements of this tool.

Conclusions: The RWR offers promise for older drivers to self-screen their ability to drive as well as provide education on safe driving, however modifications are recommended for this tool.

Key Words: $\quad$ Aging, driver, self-regulation, screening 


\section{INTRODUCTION}

Older drivers can be at an increased risk of crashing due to age-related changes in their driving abilities as well as common medical conditions (Dickerson et al., 2007). In response to declines in their abilities, many older drivers use self-regulation strategies (Ball et al., 1998; Molnar et al., 2008; Unsworth et al., 2007). However, some older adults appear to be unaware of age- or disease-related changes that may negatively affect their driving performance (Baldock et al., 2006; Freund et al., 2005; Marottoli et al., 1998; Okonkwo et al., 2008).

Self-screening tools have been developed as aids for older adults to help them learn more about their ability to drive (Eby et al., 2003). The Roadwise Review CD-ROM (RWR) is one tool that has been introduced, to allow older adults to learn more about their driving abilities, so that they can take appropriate steps to stay on the road safely (Staplin et al., 2006). As indicated by Pellerito and Schold Davis (2005), the RWR is one means of client self-screening that could form part of the driver rehabilitation process to ensure community mobility for older adults.

The RWR was released by the American and Canadian Automobile Associations in 2005 to "educate and empower older persons to do whatever they can to remain safely behind the wheel" (Staplin et al., 2006). It tests abilities related to driving such as general mobility, flexibility, vision, processing of visual information, and memory. These abilities were found to be linked to crashes in a prospective trial called the Maryland Pilot Older Driver Study (MaryPODS (Staplin et al., 2003a; Staplin et al., 2003b)). This study was conducted by licensing staff assessing the older drivers who had just renewed their licenses. They were then prospectively followed for crashes. Two other older samples (about 77 years of age on average) also had these abilities examined: a residential community sample, and a medical referral group. The RWR uses cut-points for scoring that were found to be associated with increased crash risk in MaryPODS.

The original data from MaryPODS were not obtained through computer testing, except for the useful field of view test (UFOV), but it was felt that computerizing the tests would improve standardization (Staplin et al., 2006). From this, a screening tool called the Driving Health Inventory ${ }^{\circledR}(\mathrm{DHI})$ was developed for health care practitioners (Staplin et al., 2006). The RWR was adapted from the DHI to allow older drivers themselves to go through the screening tests privately in their own homes, and then 
receive feedback, with friends or family members acting as their testing partner. In order to computerize the test for this home-based screening, some changes were made to the actual tests. For example, the Visual Search test, which is Trail Making Part B, went from a paper and pencil based test in MaryPODS to one that requires older adults to manipulate the mouse to move between the numbers and letters of the test. No testing was done by the developers to determine what effects these changes might have on the scores of older drivers, or what the responses of the older drivers would be to the feedback that is provided by the CD-ROM.

Two previous studies have been published on the DHI (Edwards et al., 2008; Scialfa et al., 2010), and its validity. Edwards et al. (2008) reported that it distinguished between younger and older drivers ( $\mathrm{n}$ $=257$, age range $=18$ to 87 years old) in their abilities related to driving across several domains, and scores were also different between older drivers who had self-reported crashes and those who did not. Scialfa et al. (2010) found that the failure rate was high for the Head/Neck Flexibility test, but that overall the $\mathrm{DHI}$ was not a good predictor of driving difficulties or self-reported collisions in the drivers 50 to 88 years old $(n=73)$ they studied. Unlike the two studies previously described, the study by Myers et al. (2008) was a process evaluation of the RWR which relied on peer partners to conduct the screening tests $(n=34)$. Several issues were found with peer testing whereby some scores were likely over- and underestimated because of this method of testing. Focus groups were held to gather information from the older drivers regarding their immediate responses to having done the RWR.

The specific purposes of the current mixed methods study were to determine: 1 ) how older drivers in two different centers would score on the RWR with trained research assistants acting as partners rather than untrained peers; 2) what factors would affect their scores; 3) how our participants would perform compared to the original MaryPODS participants upon which the RWR norms were created; 4) what our participants' immediate and longer term responses would be to the RWR; and 5) what actions or intentions would result from the session with the RWR.

\section{METHODS}

\section{Participants}

Older male and female current drivers were recruited $(n=96)$. In contrast to MaryPODS, our sample was one of convenience recruited via various means including: ads in papers, email newsletters, senior 
centers, and posters in community locations. This method of recruitment would be similar to what could be expected for the RWR, because older drivers would be choosing to use the CD-ROM for selfscreening, as compared to MaryPODS whereby the majority of participants were older drivers who were being screened because they were renewing their licenses. We chose to recruit drivers 70 years and older because the main clientele for senior driver education programs in our locations were usually in their 70 s, and this is approximately the age when the increase in fatal crashes per mile driven usually occurs (Braver et al., 2004). In two instances, we allowed wives who were in their late sixties to participate along with their husbands. The results of the various analyses performed were not changed significantly by removing these two subjects (e.g., $p$ values for t-tests comparing sites did not change the level of significance), so therefore, we have included them in the analyses presented. The protocol received ethical approval from the University of Manitoba and the University of Victoria, and all participants provided written informed consent.

\section{Procedures}

Participants completed a questionnaire to determine their demographic information, driving history and health characteristics, prior to arriving for the RWR session. Just prior to the RWR portion of the session all participants completed the Modified Mini Mental Status Examination (3MS; Teng and Chui, 1987) to assess their cognitive status, which also allowed us to provide Mini Mental Status Examination (MMSE) scores.

RWR session: To standardize the delivery of instructions between sites, the participants relied on the CD-ROM for the instructions on the tests. The tests included: Leg Strength and General Mobility (a short rapid walk test); Head/Neck Flexibility test (whereby the seated individual tries to see an object on the computer screen 10 feet directly behind them); visual acuity testing under both low and high contrast conditions; Visualizing Missing Information (which is a modified version of a sub-test of the Motor Free Visual Perception Test); Visual Information Processing Speed (sub-test 2 of the UFOV), Visual Search (Trail Making Part B), and Working Memory (3 word recall). Throughout the session, the participants could choose to listen to background information or to repeat information, instructions or practice, as allowed by the CD-ROM.

Testing proceeded as indicated on the CD-ROM, however, based on pilot testing, it was apparent 
that some additional instructions were necessary. The only departures were the following:

1. Although it was not mandatory, we specifically encouraged participants to practice using the mouse.

2. One pilot participant became quite distraught because of her perceived performance on the "Visual Information Processing Speed" (UFOV) test, which meant she was not able to perform well on the subsequent tests, and she almost stopped using the CD-ROM altogether. Therefore, additional instructions were added at both sites: "This test requires you to identify, and to note the location of objects shown on the computer screen. The objects are visible for only a brief moment; the test determines when the objects have been shown too briefly for you to see. It is expected you may 'miss' some objects. Do not become discouraged; simply make a guess, re-focus and be ready for the next objects to be shown."

Following completion of the tests, participants were given their results and an opportunity to view additional information provided by the CD-ROM related to their results on the computer screen. They then completed a questionnaire on the RWR session, providing written answers via closed and openended questions on their experience and what they might do as a result of having completed the RWR. Before leaving the session they were given feedback sheets to take with them that had the same written information as on the computer screen. The printed feedback sheet included: a summary sheet which indicated each test done; their score on the test (numerical or categorical); and their risk (if any) of crashing based on their score. In addition, participants also received information indicating: what their level of risk suggests, what they should do, and examples of where that underlying ability might be required in driving, according to what the CD-ROM had indicated based on their level of impairment. For example, if they had a mild impairment, one piece of information they received from the CD-ROM regarding "Am I at Risk?" was: "Though you appear to have a mild loss in Visual Information Processing Speed, you may still be able to drive without exposing yourself or others to any significant increase in crash risk." In answer to the question "What should I do?", individuals would be told the following for a Serious impairment: "You should schedule a visit with your physician, an occupational therapist, or 
certified driving rehabilitation specialist without delay. Ask specifically about how such a loss could affect your driving, and what you should do to stay safe. Ask a friend or relative for a ride to your appointment, or use some form of alternative transportation."

At the end of the session they were given another questionnaire to take with them and then mail back about two weeks after their session with the RWR (the response rate across both sites was 95\%). This two week time frame was given to allow the participants some time to reflect on the experience and to give them time to follow-up or start the process of following up on any recommendations.

\section{Data analysis}

All quantitative data were analysed using SPSS (SPSS Inc., Chicago, IL) and SigmaPlot (Systat, Chicago, IL). P values $<0.05$ were considered to be statistically significant. Means and standard deviations were calculated to describe participants. Independent sample t tests or Mann Whitney U tests were done to compare groups (e.g., Victoria vs Winnipeg, men vs women) for continuous or categorical data. Proportions were compared with chi-square tests, with Fisher Exact tests being used instead when the cell sizes were too small.

Binary logistic as well as Best Subsets regressions were done to explore the relationships between participant characteristics and the scores on tests. Each test was examined individually to determine which participant characteristics were related to performance. Analyses were not performed for visual acuity because of the low number of impairments that were uncovered. For tests with continuous data, both best subsets linear regression (raw score as dependent variable) and logistic regression analyses were conducted. For the logistic regression analyses, impairment was re-coded as a dichotomous variable whereby mild and serious impairments were both considered to be impaired. Age, gender and location of testing (Winnipeg vs Victoria) were used in the models for all tests. Other independent variables were selected for each test individually based on the most likely explanatory variables.

Comparisons between our participants and the MaryPODS samples were made using onesample t tests.

To examine the quantitative survey data collected after the RWR session, either immediately or after two weeks, proportions were compared with chi-square tests (Fisher Exact tests were used instead 
when the cell sizes were too small). For the small amount of qualitative data from the surveys, the seven open-ended questions were analysed by collapsing the responses into themes, and determining how frequently similar responses were made.

\section{RESULTS \\ Participants}

In total, 96 older drivers participated in Winnipeg $(n=42)$ and Victoria $(n=54)$. Participants ranged in age from 67 to 85 (75 \pm 5 ; mean $\pm S D$ ) years in Winnipeg, and from 70 to 91 (78 \pm 5$)$ years in Victoria. The participants reported driving for 30 to 71 years each (53 \pm 9 years), with a frequency of driving in the past week of 0 to 7 days ( $5 \pm 2$ days). The participants from the two sites were very similar in terms of their self-reported overall health, diagnoses of various medical conditions, rates of cataract surgeries, falls rate, medication usage, comfort with computers and software, self-reported ability to walk one mile, use of walking aids, and participation in exercise. The few differences between groups were: the participants in Victoria were significantly $(p<0.05)$ older than those in Winnipeg; the proportion of participants who were women was greater in Winnipeg ( $p<0.05 ; 74 \%$ versus $48 \%$ in Victoria); a greater proportion of participants from Victoria had completed high school ( $p<0.05 ; 96 \%$ versus $83 \%$ in Winnipeg), and the proportion of participants from Winnipeg who had taken driving courses was greater $(p<0.05 ; 76 \%$ versus $50 \%$ in Victoria). There were no significant differences between the Winnipeg and Victoria groups' scores on the MMSE $(28.9 \pm 1.2$ vs $28.7 \pm 1.4)$ or the 3 MS $(96.1 \pm 3.3$ vs $94.9 \pm 4.5)$. The range of scores for the MMSE was 24 to 30, with only one participant in Victoria having a score of 24 .

\section{RWR Impairments}

There were no significant differences between the groups in how they scored on the tests. Almost all participants were "impaired" according to RWR criteria on at least one test. As can be seen in Figure 1 the proportion of participants that had impairments varied greatly between the different tests, with very few participants having any kind of visual acuity impairment or mobility impairment, but the majority of

participants having a serious Head/Neck Flexibility impairment. Because the flexibility test was scored as pass/fail, there was only one level of impairment (serious). For Visual Search, about $75 \%$ of 
participants were found to have an impairment, with about $60 \%$ being categorized as mild impairment, and approximately $15 \%$ with a serious impairment. For Working Memory about $40 \%$ had an impairment, whereas for Visualizing Missing Information and Visual Information Processing Speed the impairment rate was about $25 \%$.

When the 90 participants who completed all tests were examined, only four participants had no impairments at all. Across the 90 participants there were 114 mild and 115 serious impairments. The maximum number of impairments per person, for these 90 participants, was $6(n=3)$ out of a possible 8 . The majority of participants had 1 to 3 impairments (74\%). Across all participants who had completed all tests, men had significantly more serious impairments than women $(1.7 \pm 1.3$ vs $1.0 \pm 1.0 ; U=726.5, p=$ $0.011, n=90$ ). There were no significant gender differences for mild or total impairments although there was a trend for the latter $(t=-1.9, D F=88, p=0.065$; men $=2.9 \pm 1.5$, women $=2.3 \pm 1.5$ total impairments).

The regression analyses revealed that although several participant characteristics were significant in the various models, the adjusted $\mathrm{R}^{2}$ values only ranged from 0.04 to 0.23 across the tests for the best models. In terms of factors, although the explanatory power was low, age was significant in the models for Visual Information Processing Speed, Visual Search, and Working Memory, with higher age resulting in poorer performance. Gender was only significant in the model for Working Memory, whereby women performed better - it was not a factor for any of the other tests (overall model adjusted $\mathrm{R}^{2}$ values $=$ 0.16 and 0.22 for impairment and scores on this test, when the other significant factors of age and location were included). Another factor of note included that those who were comfortable with computers had faster times on the Visual Search test (overall model adjusted $R^{2}=0.15$, including age as a factor). For Leg Strength and General Mobility, the use of ambulatory aids and the reporting of past falls related to impairment on this test (adjusted $R^{2}=0.20$ ). Having a reported diagnosis of a back or neck problem related to poorer performance on the Head/Neck Flexibility test, but the model was quite weak in predictive ability (adjusted $R^{2}=0.044$ ). For Visualizing Missing Information, the level of education attained was significant for both the number of incorrect responses (adjusted $R^{2}=0.15$ ) as well as the presence or absence of impairment (adjusted $R^{2}=0.0 .08$ ), with those attaining higher levels of education performing better. 


\section{Comparison with MaryPods}

Average data for the current participants and the MaryPODS samples are shown in Table 1. For most of the tests there were differences between the current participants and the samples reported in MaryPODS. In most cases the current participants performed better than the medical referral sample, except for Head/Neck Flexibility. In several cases the current participants performed worse than the license renewal or residential community samples. This was particularly true for the Head/Neck flexibility tests whereby more than half of our sample failed (63\%), but it was closer to $20 \%$ for the non-medically referred sample from MaryPODS, and only 37\% for the medically referred group from MaryPODS.

\section{Surveys on the RWR}

Overall, the feedback on the RWR, whether immediate or after two weeks, was positive. Almost all participants reported that: 1) the session made them more aware of changes that can affect driving $(91 \%) ; 2)$ they would recommend it to others (98\%); and 3) the information provided could be useful for helping older adults talk about driving concerns with their families (95\%). Most participants (81\%) reported that they would be willing to pay for this type of program, and over half reported that they discovered changes in themselves that they had not been aware of before. There were no significant differences between the groups in their responses to either the immediate or mailback survey, except for one question on the immediate survey. A significantly greater proportion of participants in Winnipeg indicated that they would "have a doctor check their seeing, thinking, or movement abilities" (62\% for Winnipeg versus $41 \%$ for Victoria, $p<0.05$ ). This difference was no longer apparent after two weeks (24\% for Winnipeg versus $28 \%$ for Victoria, NS), when asked "Now that you have attended this session, did you or are you going to have a doctor (or other health care professional) check your seeing, thinking or movement abilities".

Interestingly, more than $60 \%$ of participants reported that they would make changes to their driving, immediately following the RWR session. After two weeks, almost half of the participants reported that they had made changes. Of the participants who gave a specific written response as to what change was made, the most frequently reported changes were: 1$)$ related to flexibility $(n=21$; e.g., 
exercising to increase upper body flexibility, parking differently to avoid backing up, better shoulder checking, checking with health professional about neck flexibility) and 2) being more attentive, cautious, or careful when driving $(n=15)$.

When asked what they found "Most Useful" about the session either immediately after the RWR or two weeks later, the majority of participants at both sites mentioned that they found out something about their own abilities related to driving. Here is a quote from one participant "Tests suggested I don't think as quickly as I thought." Quite a few participants also reported that it confirmed that they did not have problems, e.g., "It confirmed my feeling that I was still competent to drive." Another common response was that they were now more aware about what is important for driving, e.g., "Reminder of head-neck flexibility, and the need to turn my whole body as I look over my shoulder."

When asked for suggestions for improvements, although a fairly large number had no suggestions, many suggestions were given related to individual tests, and several questioned the validity of some of the tests. Here is one example: "Make the testing more realistic, ex: Leg Strength Test - To walk from spot to B and back - it tells you zero re leg strength when sitting and touching brake." Suggestions for the Head/Neck Flexibility test included: increase the size of the object; make a scale for scores instead of just pass/fail; test for both sides and not just one, since they need to look both ways; and problems associated with gripping the chair with the hands should be addressed. Another common suggestion related to computer or mouse use and how this needed to be accommodated for this cohort, because of their lack of familiarity with computers. Regarding the informational nature of the CD-ROM, suggestions were made to include more examples of common mistakes or driving hazards. A few suggested that a road or simulator test would be beneficial in addition to the RWR.

In the General Comment section most participants just provided thanks, general positive comments of appreciation, or that it was interesting. Some suggested that the RWR should be required for all seniors. And some commented on their new awareness: "Found it jolts one into taking notice of where one is at with driving skills." 


\section{DISCUSSION}

\section{RWR Scores}

In this volunteer sample of older drivers, almost all participants (96\%) were found to have at least one test score on the RWR that was in the impaired category. The tests that resulted in the greatest rate of impairments were the Visual Search test (75\%) and the Head/Neck Flexibility test $(63 \%)$. Whereas very few participants had impairments on the Visual Acuity or Leg Strength and General Mobility tests, there were several subjects that did have impairments in Visualizing Missing Information, Visual Information Processing Speed and Working Memory.

The impairment levels of the RWR were determined from the original MaryPODS sample of 1,876 drivers aged 55 or older, with $45 \%$ of participants being women and 35\% being 55 to 64 years old (Staplin et al., 2003b). In the report for the research it was suggested that the lower cut-point (termed mild impairment in the RWR) indicates a need for "prevention", and that the higher cut-point (serious) indicates a need for "intervention" (Staplin et al., 2003b). For example, 80 seconds was calculated as the prevention cut-point for Visual Search (Trail Making Part B) and 180 seconds the intervention cut-point (Staplin et al., 2003b).

In our participants, about $75 \%$ were deemed to have an impairment in Visual Search (about $60 \%$ mild and $15 \%$ serious). Our participants on average were slower than both the license renewal and the residential community samples of MaryPODS, but faster than the medical referral sample. Reasons for the differences between groups could include age of the participants; our participants (mean age $=76.6$ years) were older than the license renewal (mean age $=68.3$ years) sample. However, the residential community sample was similar in age (mean age $=77.1$ years). Another difference was that MaryPODS used the original paper-based method of Trail making Part B whereas the RWR required moving the mouse quickly. The developers of the RWR did anticipate problems here. The DHI (that they also developed) includes a mandatory mouse practice session, and if the older driver cannot meet the "target localization latency threshold" there is an option for using the paper-based method or a touchscreen (Staplin et al., 2006). Touchscreen computers might be available in some settings like seniors centers, lifelong learning centers or community colleges; however, in the home, the main location of interest for the RWR, these options would be difficult or impossible to implement due to the lack of appropriate 
equipment (stop watches or touch screens). Nonetheless, having the threshold test added to the RWR could be advised due to possible false positives when using a mouse. Our regression model for time to complete visual search included familiarity with computers as a significant predictor. Also, in our study, the mouse did trouble some participants as suggested by this quote: "I do think anyone who isn't familiar with computers would be at a disadvantage. My friends who do not use a computer recoil at the thought!"

Another RWR test that resulted in a high impairment rate was the Head/Neck Flexibility test. Sixty of the 96 (63\%) participants could not identify the object on the screen behind them when they turned to look, and therefore were deemed to have a serious impairment. This is similar, albeit somewhat higher than the serious impairment rate in the younger sample studied by Scialfa et al (2010). In contrast, all samples in MaryPODS had much lower rates of failure (19 to 37\%; Staplin et al., 2003b)). In comparing the studies, the most obvious difference is that the participants in the present study were much older than the renewal sample. This youngest sample had the lowest failure rate (19\%). With age, it is known that flexibility declines, e.g., neck rotation was greater than 10 degrees lower in 80 year olds compared to 50 year olds (Youdas et al., 1992), so that could explain the higher impairment rate in our participants compared to the renewal sample. However, even the medical referral group in the original MaryPODS study only had a 37\% failure rate, and they were comparable in age to our participants (mean age $=76.8$ years). The test used in the MaryPODS study was not exactly the same test as that developed for the RWR, so perhaps that could explain the differences. Both are similar in that the driver sits in a chair and turns and looks. In MaryPODS though, the participant had a seat belt to keep the hips from moving on the seat, whereas in the RWR the participant holds the seat with their hands. Holding the seat might have limited their trunk rotation. Also, the object that the driver was looking for was different - a shape (circle, square, star, or cross) for the RWR, versus a clock in MaryPODS. It is unknown how much the shape of the object (or potential size differences) would affect someone's performance on this test. Certainly many participants were just not able to turn far enough, however, some could turn sufficiently to see the monitor but not the object. Future research could examine more about this indirect measure of neck and trunk flexibility and whether other issues such as vision (peripheral or acuity without glasses) might contribute to who passes or fails. 
This indirect flexibility test elicited a lot of feedback from the participants. Many participants reported that they had made changes related to flexibility which included exercising to improve flexibility and also changing the way in which they shoulder check or back up. However, others questioned the validity of this test. Part of the confusion over this test comes from the fact that the information provided (instructions and videos) indicates that this test is for the ability to turn and look while backing up as well as shoulder checking when changing lanes and/or merging, and checking at intersections. Many participants view these tasks as requiring exactly the same movement pattern, which only involves turning the head. In a previous study we found that many older drivers who have sufficient range of motion in their neck and trunk do not turn and look through the rear window while backing up (Porter et al., 2006), because they believe that mirror use or turning the head is sufficient. The video played right before the RWR flexibility test shows an older driver backing up the way many of our participants did in the previous study. This means that they are only glancing over the shoulder and at their mirrors. However, the flexibility test of the RWR tries to emulate the movements performed while backing up a vehicle in that much trunk rotation is also required to look out the rear window. More information and longer instructional videos could be included, to reduce confusion as well as increase the likelihood that appropriate steps will be taken, because many participants indicated that they were making changes to their driving as a result of going through the RWR. This means that the educational information provided could be as important as the screening.

On the Visualizing Missing Information test, the current participants were significantly different from all MaryPODS samples. They performed better than the medical referral group but slightly worse than the other groups. Again the difference with the renewal group may be due to age, but this is not the case with the community residential group. We found that education level affected the score on this test so our samples may have differed in this regard. Also, the tests were not identical, as the MaryPODS study used the actual visual closure items of the Motor Free Visual Perception test, whereas the RWR presented an original set of figures developed from US highway signs (Staplin et al., 2006).

The Working Memory test also used an "expanded set of furniture-fruit-apparel items" (Staplin et al., 2006). The modifications to the MVPT and memory tests were made to reduce the effects of training with the RWR on potential future screening using instruments like the clinically-based DHI (Staplin et al., 
2006). There were no significant differences between our participants and the MaryPODS samples on the memory test, except that the medical referral group had a poorer score. This could be expected because a high portion of those being referred for driving assessments would be expected to have cognitive issues (Stav et al., 2008).

On the Leg Strength and General Mobility test our sample had faster walk times than all of the MaryPODS samples. The tests were quite similar except that the participants in MaryPODS were required to follow a line over the $10 \mathrm{ft}(\approx 3 \mathrm{~m})$ course, whereas the RWR only required participants to walk to a point $10 \mathrm{ft}(\approx 3 \mathrm{~m})$ away and then back again. The fact that they needed to concentrate on a path in MaryPODS might have slowed their walking. Alternatively, there could be physical differences between our samples that are difficult to compare because health, body composition, walking aids and falls data were not available for comparison. Our regression analyses indicated that some of these variables can affect performance on this test.

Our sample also performed better on Visual Information Processing Speed (UFOV) compared to the residential community and medical referral groups (Staplin et al., 2003b). It is not surprising that the medical referral group had poorer performance due to possible cognitive issues. However, it is surprising that our participants performed better than the residential community sample of approximately the same age, since age was the only explanatory factor for this test in our sample. Other possible explanations include: our additional instructions, mode of responding (mouse vs light pen), and education backgrounds (unknown for the residential community sample). When comparing our participants' results to normative data based on a much larger sample $(n=2,759)$ where age and education (albeit to a small degree) affected scores (Edwards et al., 2006), our participants would fall somewhere between the "< 12 years education" and "= 12 year education" for the $75-79$ age category.

Three other studies have published data using the DHI (Edwards et al., 2008; Scialfa et al., 2010) and the RWR (Myers et al., 2008). The latter study was a companion study to the one reported here. It was a process evaluation that involved peer (untrained senior) partners instead of research assistants (Myers et al., 2008). One of the former studies was conducted in a similar fashion to ours, in that a peer partner was not involved (Scialfa et al., 2010). Similar scores were seen between the DHI sample $(n=$ $119, \geq 65$ years old) and ours in many tests. Our sample performed better on leg strength, whereas the 
DHI sample was better on visual information processing. Differences between our samples could likely be accounted for by age, health status, or other demographic factors, but detailed information was not available to make these comparisons.

When compared to the group that performed the tests with peer partners $(n=34)$ (Myers et al., 2008), large differences in the rates of impairment are evident for Head/Neck Flexibility and Leg Strength and General Mobility. In our study only $12 \%$ of participants were found to have an impairment for Leg Strength and General Mobility. In contrast, in the peer partner study $47 \%$ of participants had an impairment. There were no apparent differences between the groups in their descriptive characteristics (e.g., self-reported health or ambulatory aid use) that could explain the discrepancy in these impairment rates. However, it was suggested for the peer partner study, that the validity of this test was hampered by the skill of the partner in being able to operate the timer buttons properly with the mouse. This resulted in slower times being recorded than were actually performed, and hence could have placed them into the impaired category. For the Head/Neck Flexibility test, partners occasionally indicated that the participant had identified the object correctly, when in fact they had not, which may explain why the peer partner participants had a lower rate of impairment for this test ( $41 \%$ vs $63 \%$ in the current study). The effect of peer partners on scores or impairments was not examined in the original development of the RWR from the MaryPODS' protocol. This is important because MaryPODS was conducted with trained licensing staff and not peer partners, but the same criteria for impairments are being used for the RWR.

\section{Responses to the RWR}

Generally the responses to the RWR were very positive. Almost all participants found it useful, were more aware of changes that could affect driving, and would recommend it to friends or family. Well over half had conversations with friends and family about their driving, and just under half reported making changes regarding their driving. These changes included behaviors to improve functional performance related to driving as well as actual driving behaviors. A smaller percentage of participants reported consulting (or planning to consult) a health care professional about their abilities related to driving. In one specific case, a visual acuity impairment score did prompt a health care visit by one participant which ultimately resulted in cataract surgery and a great improvement in vision. 
To increase the likelihood that older drivers take additional steps toward more detailed assessments and possible interventions after the RWR, there certainly needs to be a level of trust in the tests themselves. Several participants indicated that they had issues with the validity of the tests, which may partly explain why only about $25 \%$ of participants had already consulted or were planning to consult a health care professional, even though almost all participants had at least one impairment, and therefore were recommended to visit a health care professional by the RWR. Other factors that could explain the lack of participant action in this regard include: 1) fear over possible loss of license, or 2) participants were not prepared for making any changes or taking action because they felt they were perfectly fit to drive before completing the RWR. Here is a quote from one participant that illustrates the latter: "Slightly humbling experience, I had expected to float through with perfect scores on all points". A participant like this might not have been ready to take any steps regarding his driving. As Stalvey and Owsley (2003) point out, driver interventions need to consider several educational theories, including the transtheoretical model of behavior change which proposes that there are stages of readiness for change (Prochaska et al., 1997). A driver like the one quoted above may have been in the pre-contemplation stage prior to the RWR and therefore would not be as likely to act on RWR recommendations.

The limitations of this study include the small sample size limited to Canadian drivers, and the fact that we only followed the participants for two weeks. More research on the RWR could be done to examine the longer term responses of larger samples of older drivers across many jurisdictions to their scores and the feedback provided. This could include investigating what actions they might take within the months after utilizing such a tool. Also, the impact of the RWR on driving patterns and future crashes should be explored. This would be true for those who have been told that they have impairments and might self-regulate more, as well as those who might become over-confident because they have been given a message that they have no impairments and are therefore, are at minimal risk of crashing.

In conclusion, based on participant reporting the RWR was found to be a useful tool, elicited conversations about driving, and almost half of the participants reported making changes in their driving. Only a quarter reported consulting or planning to consult a health care professional, even though almost all participants were found to have at least one impairment. Recommendations for improvements to the RWR, include adding a threshold test for mouse performance, improving the presentation of some driving 
tasks (e.g., backing up), adding more instructions, toning down the feedback on consulting health care providers, and providing additional educational elements related to driving. Future research could investigate more about the tests that were modified for the purposes of the RWR (e.g., Head/Neck Flexibility test, Visual Search), and also explore what factors lead RWR users to make changes in their driving or to consult others, to help them to stay on the road as safe drivers and enable their community mobility.

\section{ACKNOWLEDGMENTS}

Support for this project was received from the Canadian Institutes of Health Research Institute of Aging. Additional support was provided by the Michael Smith Foundation for Health Research award for research unit infrastructure held by the Centre on Aging at the University of Victoria. A research personnel award from the Canadian Institutes of Health Research, Institute of Aging provided support for Holly Tuokko. A Research Fellowship from the Health, Leisure and Human Performance Research Institute provided support for Michelle Porter. The authors would also like to acknowledge Gerry Giesbrecht, Kristina Kowalski and Linda Johnson for assistance with testing; Good Neighbours Seniors Centre and Creative Retirement for providing space for RWR sessions; and the other members of the MOBILE team: Michel Bédard and Anita Myers. 


\section{REFERENCES}

Baldock, MRJ, Mathias, JL, McLean, AJ, Berndt, A. Self-regulation of driving and its relationship to driving ability among older adults. Acc Anal Prev. 2006;38:1038-1045.

Ball, K, Owsley, C, Stalvey, B, Roenker, DL, Sloane, ME, Graves, M. Driving avoidance and functional impairment in older drivers. Acc Anal Prev. 1998;30:313-322.

Braver, ER, Trempel, RE. Are older drivers actually at higher risk of involvement in collisions resulting in deaths or non-fatal injuries among their passengers and other road users? Inj Prev. 2004;10:2732.

Dickerson, AE, Molnar, LJ, Eby, DW, Adler, G, Bedard, M, Berg-Weger, M, Classen, S, Foley, D, Horowitz, A, Kerschner, H, Page, O, Silverstein, NM, Staplin, L, Trujillo, L. Transportation and aging: a research agenda for advancing safe mobility. Gerontologist. 2007;47:578-590.

Eby, DW, Molnar, LJ, Shope, JT, Vivoda, JM, Fordyce, TA. Improving older driver knowledge and selfawareness through self-assessment: the driving decisions workbook. J Safety Res. 2003;34:371381.

Edwards, JD, Leonard, KM, Lunsman, M, Dodson, J, Bradley, S, Myers, CA, Hubble, B. Acceptability and validity of older driver screening with the DrivingHealth Inventory. Acc Anal Prev. 2008;40:11571163.

Edwards, JD, Ross, LA, Wadley, VG, Clay, OJ, Crowe, M, Roenker, DL, Ball, KK. The Useful Field of View Test: Normative Data for Older Adults. Clin Neuropysc. 2006;21:275-286.

Freund, B., Colgrove, LAA, Burke, BL, McLeod, R. Self-rated driving performance among elderly drivers referred for driving evaluation. Acc Anal Prev. 2005;37:613-618.

Marottoli, RA, Richardson, ED. Confidence in, and self-rating of, driving ability among older drivers. Acc Anal Prev. 1998;30:331-336. 
Molnar, LJ, Eby, DW. The relationship between self-regulation and driving-related abilities in older drivers: an exploratory study. Traffic Inj Prev. 2008;9:314-319.

Myers, AM, Blanchard, RA, Macdonald, L, Porter, MM. Process evaluation of the American Automobile Association Roadwise Review CD-ROM - Observed and reported experiences of older drivers. Topics Geriatr Rehab. 2008;24:224-238.

Okonkwo, OC, Crowe, M, Wadley, VG, Ball, K. Visual attention and self-regulation of driving among older adults. Int Psychogeriatr. 2008;20:162-173.

Pellerito, JM, Schold Davis, E. Screening driving and community mobility status: A critical link to participation and productive living. OT Practice. 2005;10:9-14.

Porter, MM, Conci, SJ, Huebner, KD, Ogborn, DI. Are rear checking behaviors determined by range of motion in older drivers? Transportation Research Board Annual Meeting. 2006;0972.

Prochaska, JO, Velicer, WF. The transtheoretical model of health behavior change. Am J Health Promot. $1997 ; 12: 38-48$.

Scialfa, C, Ference, J, Boone, J, Tay, R, Hudson, C. Predicting older adults' driving difficulties using the Roadwise Review. J Gerontol B Psychol Sci Soc Sci. 2010;65:434-437.

Stalvey, BT, Owsley, C. The development and efficacy of a theory-based educational curriculum to promote self-regulation among high-risk older drivers. Health Promot Pract. 2003;4:109-119.

Staplin, L, Dinh-Zarr, TB. Promoting rehabilitation of safe driving abilities through computer-based clinical and personal screening techniques. Topics Geriatr Rehab. 2006;22:129-138.

Staplin, L, Gish, KW, Wagner, EK. MaryPODS revisited: updated crash analysis and implications for screening program implementation. J Safety Res. 2003a;34:389-397.

Staplin, L, Lococo, K, Gish, K, Decina, L. Model driver screening and evaluation program final technical report, Volume 2: Maryland Pilot Older Driver Study. 2003. 
Stav, WB, Justiss, MD, McCarthy, DP, Mann, WC, Lanford, DN. Predictability of clinical assessments for driving performance. J Safety Res. 2008;39:1-7.

Teng, EL, Chui, HC. The Modified Mini-Mental State (3MS) examination. J Clin Psychiatry. 1987;48:314-318.

Unsworth, CA, Wells, Y, Browning, C, Thomas, SA, Kendig, H. To continue, modify or relinquish driving: findings from a longitudinal study of healthy ageing. Gerontology. 2007;53:423-431.

Youdas, JW, Garrett, TR, Suman, VJ, Bogard, CL, Hallman, HO, Carey, JR. Normal range of motion of the cervical spine: an initial goniometric study. Phys Ther. 1992;72:770-780. 
TABLE 1 Comparison of the current sample to the MaryPODS samples

\begin{tabular}{|c|c|c|c|c|}
\hline Test & Current study & License Renewal & $\begin{array}{l}\text { Residential } \\
\text { Community }\end{array}$ & Medical Referral \\
\hline $\begin{array}{l}\text { Leg Strength and } \\
\text { General Mobility (s) }\end{array}$ & $6.2 \pm 1.2$ & $6.4 \pm 1.9^{*}$ & $6.8 \pm 1.9^{*}$ & $7.8 \pm 2.4^{*}$ \\
\hline $\begin{array}{l}\text { Head/Neck Flexibility (\% } \\
\text { failed) }\end{array}$ & 63 & 19 & 23 & 37 \\
\hline $\begin{array}{l}\text { Visualizing Missing } \\
\text { Information (\#) }\end{array}$ & $2.2 \pm 1.8$ & $1.7 \pm 1.8^{*}$ & $1.6 \pm 1.7^{*}$ & $2.7 \pm 2.3^{*}$ \\
\hline $\begin{array}{l}\text { Visual Information } \\
\text { Processing Speed (ms) }\end{array}$ & $182.7 \pm 107.3$ & $173.1 \pm 155.0$ & $220.0 \pm 158.4^{*}$ & $321.8 \pm 179.5^{\star}$ \\
\hline Visual Search (s) & $124.8 \pm 59.2$ & $106.6 \pm 47.7^{*}$ & $110.2 \pm 50.2^{*}$ & $170.4 \pm 72.7^{*}$ \\
\hline Working Memory (\#) & $0.5 \pm 0.8$ & $0.6 \pm 0.9$ & $0.6 \pm 0.8$ & $0.9 \pm 1.0^{*}$ \\
\hline
\end{tabular}

Note: ${ }^{*} p<0.05$ significantly different from the current study based on a one sample t test. 


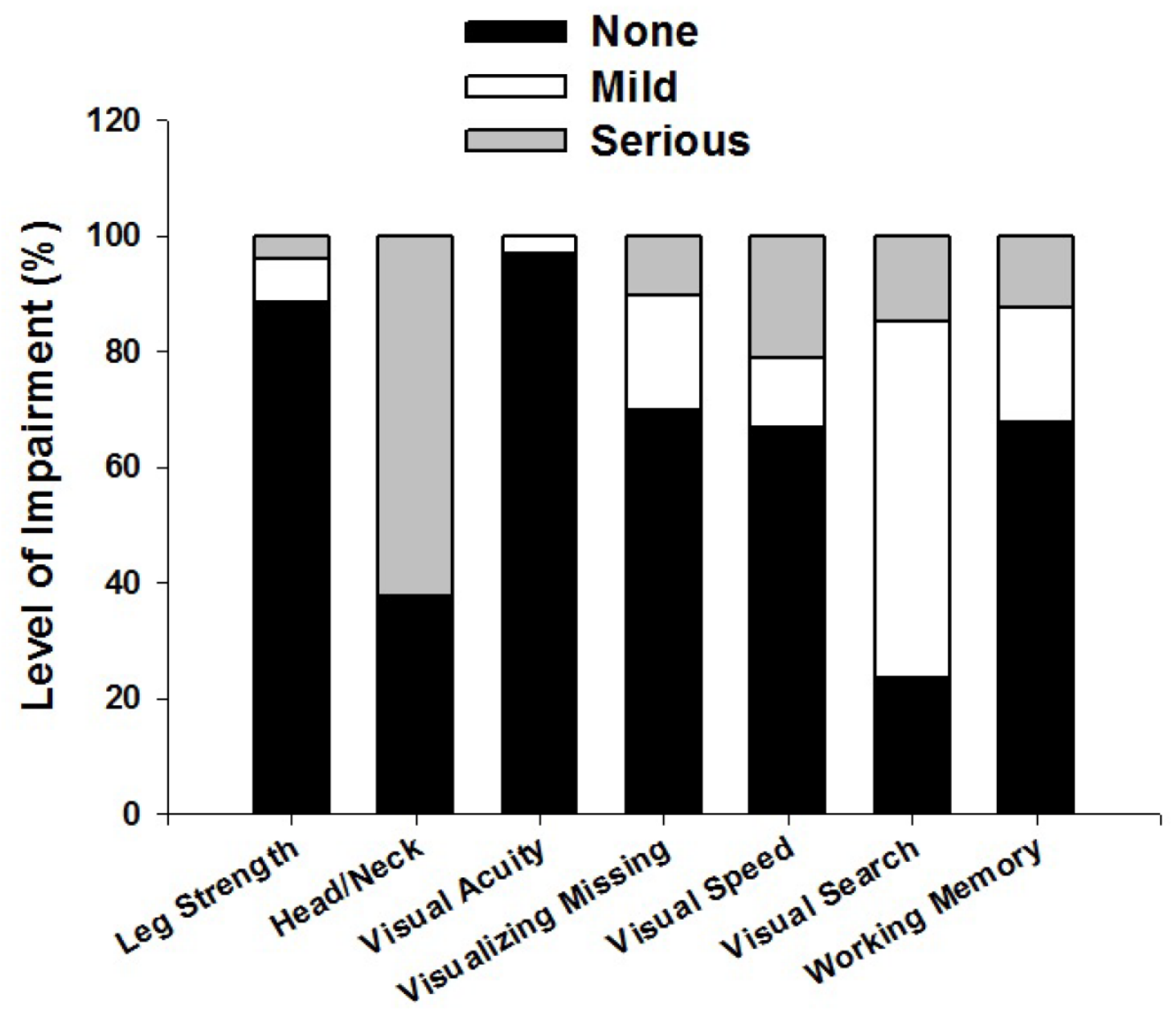

FIGURE 1. Percentage of participants at each level of impairment is shown for each of the screening tests. Leg strength = Leg Strength and General Mobility, Head/Neck = Head/Neck Flexibility, Visualizing Missing = Visualizing Missing Information, Visual Speed = Visual Information Processing Speed. 